\title{
Asymmetry of the perceptual span in reading
}

\author{
GEORGE W. McCONKIE \\ Cornell University, Ithaca, New York 14853 \\ and \\ KEITH RAYNER \\ University of Rochester, Rochester, New York 14627
}

\begin{abstract}
An on-line computer technique was used to determine whether three skilled readers acquired visual information equally far to the left and right of central vision during fixations in reading. None of the subjects appeared to use visual information more than four character positions to the left of the fixation point (smaller distances were not tested), though all of them acquired visual information substantially further than that to the right. Thus, the region of useful visual information in reading is asymmetric around the fixation point.
\end{abstract}

Recent research has found that readers acquire visual information from a relatively small region of text during a fixation as they read (McConkie \& Rayner, 1975; Rayner, 1975). This experiment investigated whether visual information is acquired from a symmetrical region around the center of vision or whether the region tends to be asymmetrical.

Studies of word identification with tachistoscopic presentation have found that words can typically be identified further to one side of the fixation point than to the other. For instance, Bouma (1973) reports that words can be identified with $60 \%$ accuracy when they begin $2 \frac{1}{2}$ deg to the right and when they end $11 / 2$ deg to the left of the fixation point, thus yielding an asymmetry of a full degree of visual angle.

The present study investigated the perceptual span of people reading text, using the technique described by McConkie and Rayner (1975). An eye-movementcontrolled display system allowed a computer to monitor the reader's eye position as he read from a computer-generated text display, and made it possible to modify the text display on the basis of the reader's eye position. Initially, an unreadable pattern (every letter of the original text had been replaced by another letter) was displayed on a cathode ray tube (CRT).

The research described in this report was carried out at the Artificial Intelligence Laboratory at the Massachusetts Institute of Technology. The authors wish to express their gratitude to Dr. Marvin Minsky and Mr. Russell Noftsker for the use of the extensive computer facilities at the AI Lab, and to David Silver and Gary Wolverton for assistance with programming and data analysis. The research was supported by a special fellowship from the National Institute of Mental Health to the first author and by Grant OEG 2-71-0531 from the Office of Education. Portions of these data were presented at the 1975 meetings of the American Educational Research Association. Reprints may be obtained by writing to George W. McConkie, 214 Stone Hall, Cornell University, Ithaca, New York 14853. This paper is sponsored by Ralph N. Haber, who takes full editorial responsibility for its content.
However, when the reader fixated the first line of text, the display was immediately modified by replacing letters within a certain region around the fixation point on that line with the corresponding letters from the original text. This created a region of normal text for the reader to see on that fixation. This region will be called the window. When the reader made a saccadic movement, the text in this window area returned to its original, unreadable form, and a new window of normal text was created at the location of the next fixation. Thus, wherever the reader looked, he could see normal text and read quite normally. However, the experimenter could determine the size and location of that region with respect to the reader's fixation point.

McConkie and Rayner (1975) previously used this technique to determine how small the window must be before characteristics of the text pattern outside the window began to affect reading performance, and to determine how far from the center of vision the reader acquires visual information of various types. However, in that study, the window always extended the same distance to the left and right of the fixation point. In the present study, the window was shifted with respect to the fixation point, extending further to the left or right, in order to determine whether visual information used in reading tends to be acquired further to the left or right of the fixation point.

\section{METHOD}

\section{Subjects}

Three high school students who had been identified as being among the best readers at their school served as subjects. All had participated in previous studies and had at least $8 \mathrm{~h}$ of practice reading under computer eye-monitoring conditions. They were paid a base rate for their participation plus a bonus based on their performance on test questions.

\section{Materials and Design}

Six passages of approximately 500 words each were extracted 


\author{
1 Original Text \\ 2 Displayed Line \\ 3 Centered Window \\ 4 Right-Shifted Window \\ 5 Left-Shifted Window
}

Graphology means personality diagnosis from hand writing. This is a
Cnojkaiazp wsorc jsncaroilfp bloqraele tnaw kori mnlflrz. Ykle le o
Cnojkaiazy means personality diagnosis from hand wnlflrz. Ykle le o
Cnojkaiazp wsorc jsncaroiity diagnosis from hand wnlflrz. Ykle le o
Cnojkaiazy means personality diagnaele tnaw kori mnlflrz. Ykle le o

Figure 1. Examples of the types of displays seen by the readers. Line 1 shows a line of original text. Line 2 shows the mutilated version of the text which was displayed on the cathode-ray tube. Lines 3-5 present examples of the appearance of that line under the different experimental conditions if a subject's center of vision was on the letter " $\mathrm{d}$ " in "diagnosis." The window area is underlined here, but, of course, no such marking occurred in the study. The display actually contained about eight lines of text.

from a high school psychology text. None of the subjects had taken a psychology course. Each passage was divided into six pages for presentation.

The two independent variables used in the study were window condition and peripheral text pattern. Three window conditions were used. In the first (centered window condition), the window was 41 character positions in width and was centered with respect to the point of fixation: That is, the letter identified as being in the center of vision and the 20 character positions to each side contained normal text. In the second window condition (left-shifted window), a 25-character position window was used which was shifted 8 character positions to the left with respect to the fixation position: That is, the window included the character in the center of vision, 20 character positions to the left, and only 4 to the right. In the third condition (the right-shifted window), a 25-character position window was shifted 8 character positions to the right; thus, the window included the character in the center of vision, 20 character positions to the right, and only 4 to the left. An example of each condition is shown in Figure 1.

The peripheral text pattern variable refers to the type of text pattern existing in the region outside the window. Two peripheral text patterns were used, both produced by replacing letters in the text with visually similar letters. Visual similarity was determined from confusability matrices published by Bouma (1971) and Hodge (1962). In one pattern, called the spaces pattern, the spaces and punctuation marks were not changed. In the other, called the filled pattern, spaces and punctuation marks were each replaced by a letter, thus removing all information about word lengths and beginnings and ends of sentences.

The factorial combination of three window conditions and two peripheral text patterns yielded six experimental conditions.

Each subject read all six passages, a total of 36 pages, with 6 pages of text in each experimental condition. Window condition remained constant for a subject through all pages of a passage, but peripheral text pattern alternated from page to page. Window condition was counterbalanced over passages.

\section{Apparatus and Procedure}

The apparatus used in this research has been described by McConkie and Rayner (1975) and is similar to a system described by Reder (1973). A Bicmetrics Model SG eye-movement monitor was interfaced to a Digital Equipment Corporation (DEC) PDP-6 computer, thus permitting on-line recording of eye movements. The computer controlled a DEC Model 340 CRT which had a character generator for upper- and lowercase letters. The CRT had a displayable area of $21 \times 18.5 \mathrm{~cm}$. capable of displaying 40 lines of 80 characters each. The subject was $53 \mathrm{~cm}$ from the CRT, so a 72-character line occupied about $18 \mathrm{deg}$ of visual angle, or 4 letters/deg. Only 8 to 10 double-spaced lines of text were displayed at once. A bite bar was used to reduce head movement.

Prior to reading each passage, each subject performed a calibration task which involved looking directly at a series of points on the CRT, while the computer obtained the conversion values it needed to determine eye position from the eye-movement monitor signal. The first and successive pages of text appeared on the CRT when the subject pressed a button. When he had finished reading the passage, he was given a multiple-choice test on the passage. The test was scored, and the subject was given the results prior to reading the next passage. Subjects received a bonus of 1 cent for each correct answer. Data for each subject were collected in a single session.

\section{RESULTS}

A number of scores were obtained from the eye-movement records for each page of text read by each subject. These included 1st, 2nd, and $3 \mathrm{rd}$ quartiles for fixation durations, 1st, 2nd, and 3rd quartiles for saccade lengths, number of regressions, median durations of fixations in regressions, median durations of saccadic movements, and total reading
Figure 2. Effect of window condition on median length of forward saccades for the three subjects, plus 1st and 3rd quartiles.
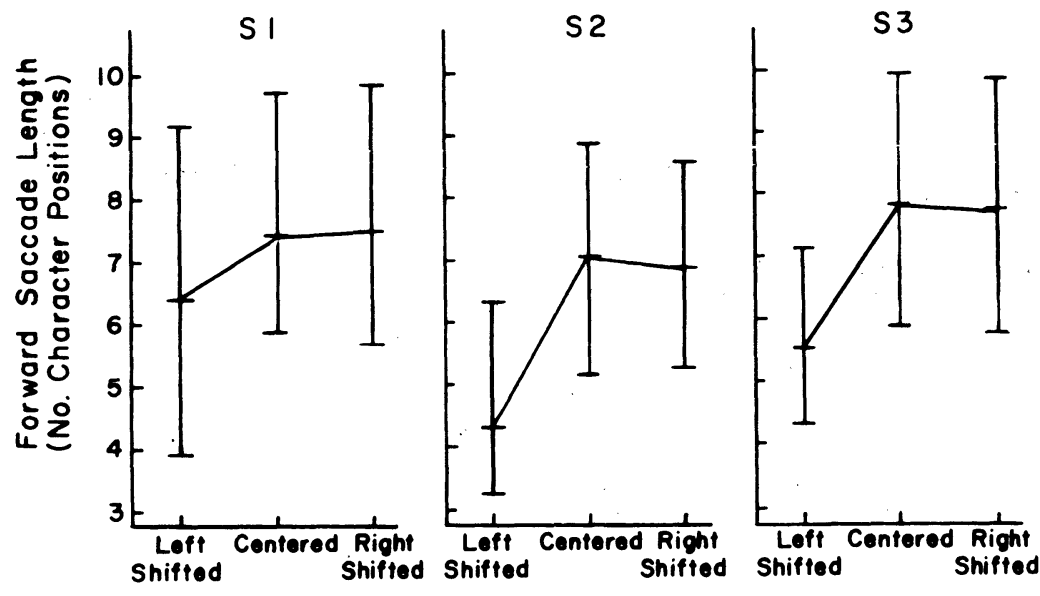

Window Position 

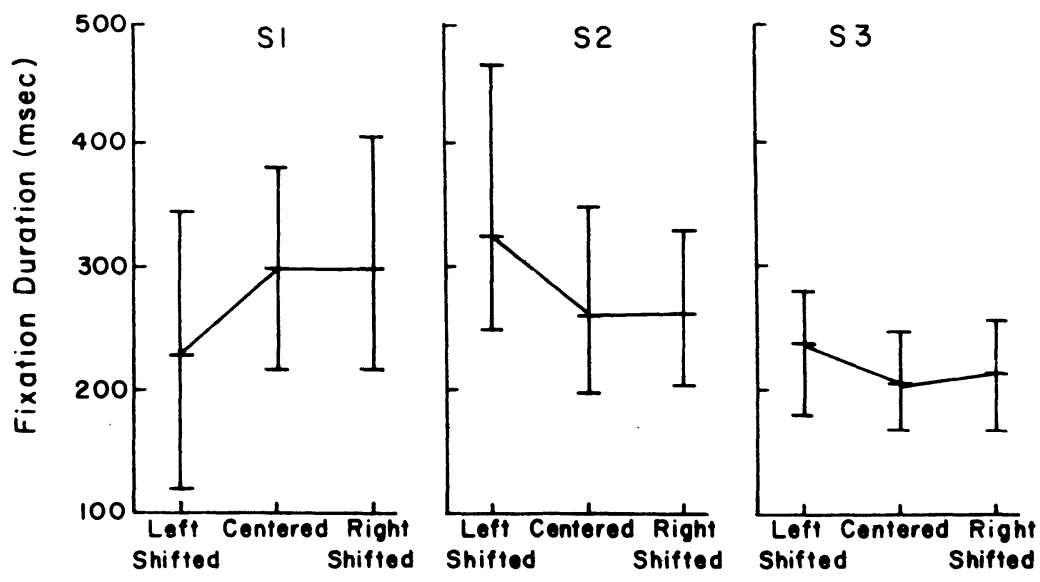

Figure 3. Effect of window condition on median fixation duration for the three subjects, plus 1st and 3rd quartiles.

Window Position

time per 100 characters. Each of the variables was subjected to a three-way analysis of variance, with factors being Window Condition, Peripheral Text Pattern, and Subjects. Main effects due to Subjects and Subject by Window interactions were significant in every analysis, usually at the .0001 level. The main effect of Window Condition was significant in three of the analyses at the .05 level.

The general pattern of the data can be summarized as follows. For every dependent variable, the centered window and right-shifted window conditions were almost identical in their data values, while the left-shifted window was very different. The data pattern for two subjects was consistently quite similar; the third subject had extreme difficulty reading under the left-shifted window condition and showed a different data pattern under this condition, including a large number of regressions, reading time which quadrupled (as compared to $24 \%$ and $28 \%$ increases for the other subjects), and fixation durations which shortened rather than increased, as with the other subjects. Figures 2, 3, and 4 present the data for lengths of saccades, durations of fixations, and number of regressive movements, plotted separately for subjects. Results of the analyses of variance for these variables are shown in Table 1.

\section{DISCUSSION}

The results of this experiment demonstrate a substantial asymmetry in the reader's perceptual span. The absence of normal text five or more character positions to the left of the center of vision produced little or no effect on the subjects' reading behavior. Removal of normal text in the same region to the right of the center of vision substantially changed the reading patterns for all subjects, although the nature of the changes produced were not consistent for all subjects. There is no evidence that the subjects acquired useful visual information more than four character positions to the left of the center of vision ( $1 \mathrm{deg}$ of visual angle), and it is possible that they did not obtain visual information even that far to the left, a possibility that requires further study. It appears that these subjects were using only visual information within the region of central foveal vision and to the right of it as they read.
The data from the present study are also interesting in response to the question of whether readers have flexibility in being able to base their reading on visual information extracted from different retinal areas. Although the same size region of normal text was available in the left-shifted and right-shifted conditions, and both were offset the same amount (although in different directions), subjects were able to read normally in one condition but not in the other. Thus, subjects do not seem to have a great deal of this sort of flexibility. Whether this is due to the establishment of reading habits, which can be modified with experience, or whether it is more basic to the reading processes is a question which requires further study.

No significant effects involving type of peripheral text pattern were found in the analyses of variance. However, McConkie and

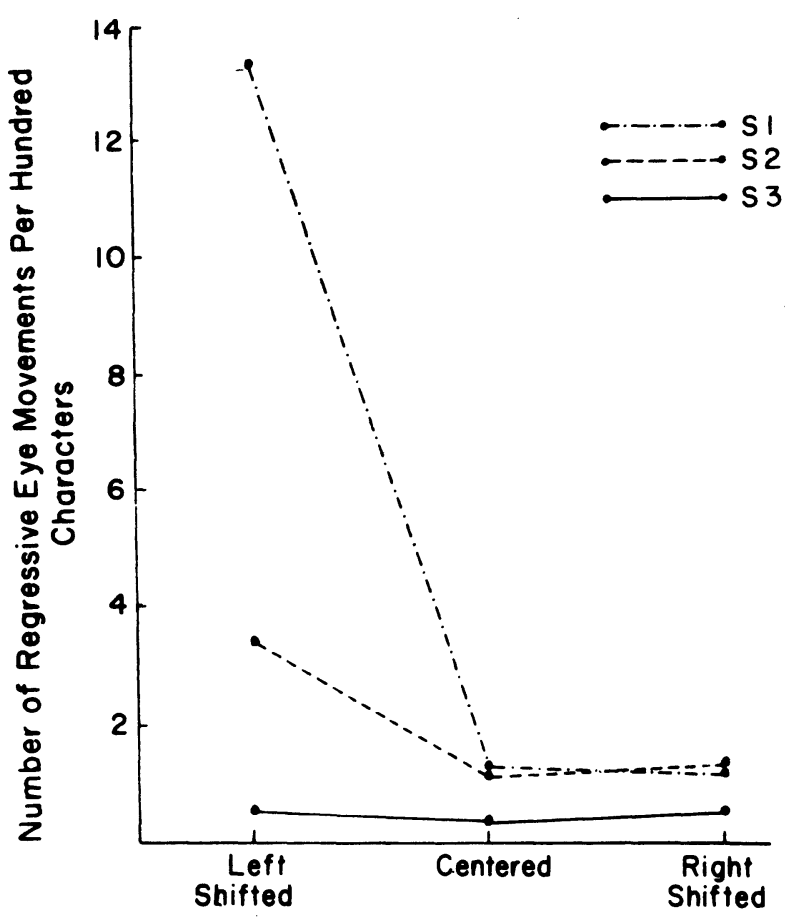

Window Position

Figure 4. Effect of window condition on the number of regressive eye movements for individual subjects. 
Table 1

Results of Analyses of Variance for Data on Lengths of Saccades, Durations of Fixations, and Number of Regressions

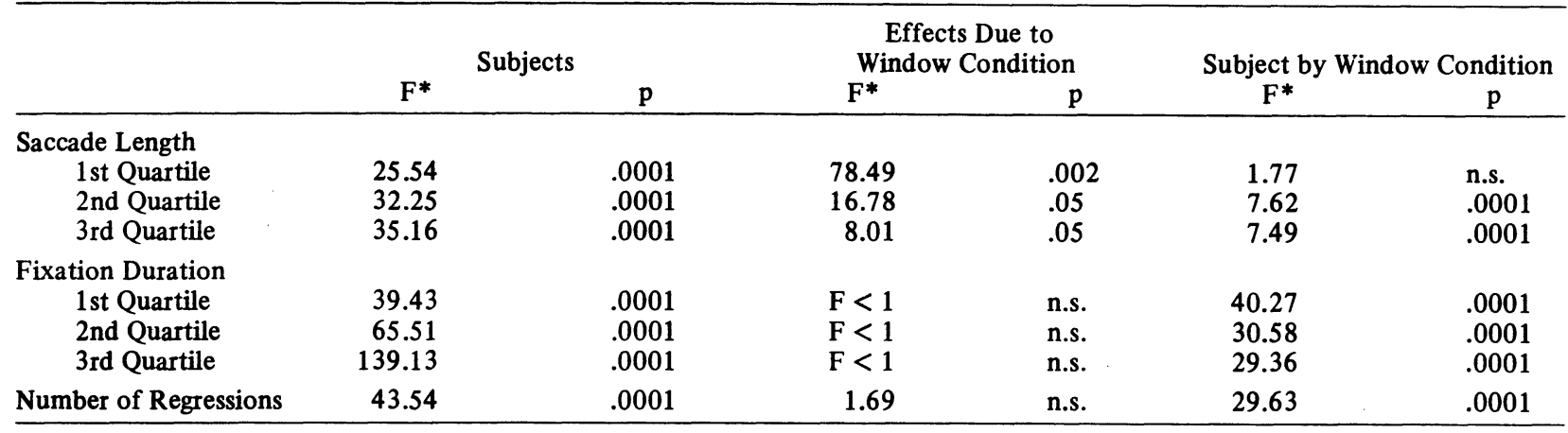

*Degrees of freedom: subjects effect, 2/90; Window condition, 2/4; Subject by Window Condition Interaction 4/90.

Rayner (1975) earlier reported that, with windows extending up to 12 letter positions to the right of the fixation point, filled conditions produced fewer long saccades than did space conditions. This effect was particularly noticeable in the 3rd quartile data. It would not be expected, then, that the centered and right-shifted window conditions in the present study would show any effect of peripheral text pattern, since they extended more than 12 character positions to the right. However, the left-shifted window condition would be expected to show an effect, since it extended only four character positions to the right. In order to determine whether this data pattern existed in the present study, saccade length data for the left-shifted window condition were further examined. As in the earlier study, saccades were found to be shorter under the filled condition, with 1st, 2 nd, and 3rd quartile values of 4.07, 5.63, and 7.52 as compared with $4.14,5.95$, and 8.27 for the spaces condition. Only the 3rd quartile data showed a significant difference $[F(1,30)=5.08, p<.05]$. Thus, the data are consistent with the earlier study in showing that saccades tend to be shorter when word length information is not present in parafoveal and peripheral vision. It should also be noted that the lack of an effect of peripheral text pattern for the right-shifted window is further support for asymmetry of the perceptual span.

Finally, the results of the present study indicates that skilled readers primarily use visual information to the right of the center of vision in reading. Further study is needed to determine whether at certain times, such as on the fixation following the return sweep of the eye as it proceeds to the next line of text, visual information to the left might become more important. These results may also have implications for the study of cortex hemispheric specialization.

\section{REFERENCES}

Bouma, H. Visual recognition of isolated lowercase letters. Vision Research, 1971, 11, 459-474.

Bouma. H. Visual interference in the parafoveal recognition of initial and final letters of words. Vision Research, 1973, 13. $767-782$.

HoDGE, D. C. Legibility of a uniform-stroke width alphabet: 1. Relative legibility of upper and lower-class letters. Journal of Engineering Psychology, 1962, 1, 34-46.

McConme, G. W., \& RAYNeR, K. The span of the effective stimulus during a fixation in reading. Perception \& Psychophysics, 1975, 17, 578-586.

RAYNER, K. The perceptual span and peripheral cues in reading. Cognitive Psychology, 1975, 7, 65-81.

REDER, S. M. On-line monitoring of eye position signals in contingent and noncontingent paradigms. Behavior Research Methods \& Instrumentation. 1973. 5. 218-228.

(Received for publication July 16, 1976.) 